This is the post peer-review accepted manuscript of:

A. Elzanaty, A. Giorgetti, and M. Chiani, "Weak RIC Analysis of Finite Gaussian Matrices for Joint Sparse Recovery," IEEE Signal Processing Letters, vol. 24, no. 10, pp. 1473-1477, Oct. 2017. The published version is available online at:

https://doi.org/10.1109/LSP.2017.2729022

(C) 2017 IEEE. Personal use of this material is permitted. Permission from IEEE must be obtained for all other uses, in any current or future media, including reprinting/republishing this material for advertising or promotional purposes, creating new collective works, for resale or redistribution to servers or lists, or reuse of any copyrighted component of this work in other works. 


\title{
Weak RIC Analysis of Finite Gaussian Matrices for Joint Sparse Recovery
}

\author{
Ahmed Elzanaty, Student Member, IEEE, Andrea Giorgetti, Senior Member, IEEE, and \\ Marco Chiani, Fellow, IEEE
}

\begin{abstract}
This letter provides tight upper bounds on the weak restricted isometry constant for compressed sensing with finite Gaussian measurement matrices. The bounds are used to develop a unified framework for the guaranteed recovery assessment of jointly sparse matrices from multiple measurement vectors. The analysis is based on the exact distribution of the extreme singular values of Gaussian matrices. Several joint sparse reconstruction algorithms are analytically compared in terms of the maximum support cardinality ensuring signal recovery, i.e., mixed norm minimization, MUSIC, and OSMP based algorithms.

Index Terms-Compressed sensing, weak restricted isometry constants, multiple measurement vectors, joint sparse recovery, row sparse matrices, mixed norm minimization, MUSIC, OSMP.
\end{abstract}

\section{INTRODUCTION}

Compressed sensing (CS) [1]-[13] for multiple measurement vectors (MMV) is a signal processing technique for efficiently recovering an $s$-row sparse matrix $\mathbf{X} \in \mathbb{R}^{n \times \ell}$ with support $S$ (i.e., only $s \ll n$ rows of $\mathbf{X}$ indexed by $S$ are nonzero) from $\mathbf{Y} \in \mathbb{R}^{m \times \ell}$ linear measurements

$$
\mathbf{Y}=\mathbf{A X}
$$

where $\mathbf{A} \in \mathbb{R}^{m \times n}$ is the measurement matrix and $m<n$ [14]-[27]. There exists a unique $s$-row sparse matrix satisfying (1) under the sufficient and necessary condition $m \geq 2 s+1-\operatorname{rank}(\mathbf{X})$ [25]. This matrix can be found by minimizing the $\ell_{0}$ quasi-norm of $\mathbf{X}$, but this problem has been shown to be NP-hard [28]. Nevertheless, for the special full rank case with $\ell \geq s, \mathbf{X}$ can be recovered from the same minimum sufficient measurements, $s+1$, via the polynomial time algorithm MUSIC [18], [29], [30].

Regarding the rank defective case $\ell<s, \mathbf{X}$ can be uniquely recovered, from a larger number of measurements, using greedy algorithms [18]-[24] or via the following mixed $\ell_{2,1^{-}}$ minimization program

$$
\text { minimize }\|\mathbf{X}\|_{2,1} \quad \text { subject to } \quad \mathbf{A X}=\mathbf{Y}
$$

where $\|\mathbf{X}\|_{2,1}=\sum_{j=1}^{n}\left\|\mathbf{x}^{j}\right\|$ with $\left\|\mathbf{x}^{j}\right\|$ indicating the $\ell_{2}$ norm of the $j$ th row of $\mathbf{X}$ [15]-[17]. Sufficient conditions for joint sparse recovery in terms of the restricted isometry constant (RIC) are provided in [23], [24]. The RIC indicates how well a linear transformation preserves distances between sparse vectors [1], [7]-[11]. ${ }^{1}$ Unfortunately, the RIC based

Copyright (c) 2017 IEEE. Personal use of this material is permitted. However, permission to use this material for any other purposes must be obtained from the IEEE by sending a request to pubs-permissions@ieee.org.

This work was supported by the European Commission under the EUMETALIC II project and by H2020 EuroCPS project (grant no. 644090).

The authors are with the DEI, University of Bologna, Via Venezia 52, Cesena, ITALY (\{ahmed.elzanaty, andrea.giorgetti, marco.chiani\}@unibo.it).

${ }^{1}$ The RIC has been analyzed for asymptotically large matrices in [7], [8] using the bound on the eigenvalues distribution provided in [31]. approach provides a worst case analysis, which does not depend on $\ell$. Therefore, it cannot explain the advantage due to the availability of multiple measurements. Instead, an average case guarantee was proposed in [17], where the probability of recovery failure is exponentially small in $\ell$ when a condition on the so-called weak restricted isometry constant (WRIC) is satisfied. For instance, considering $\mathbf{A}_{S}$ as the submatrix of A with columns indexed by the matrix support $S$, the WRIC tells how close is the column space of $\mathbf{A}_{S}$ to that spanned by another disjoint set of columns with cardinality $r$ [6]. ${ }^{2}$

In fact, sufficient recovery conditions for various reconstruction methods are based on the WRIC, e.g., $\ell_{2,1}$-minimization [17], SA-Music [18], and OSMP [19]. The WRIC for Gaussian matrices has been bounded using concentration of measure inequalities and the union bound [17]-[19]. However, this approach results in a large overestimation of the WRIC leading to an underestimation of the maximum achievable $s$.

This letter provides a probabilistic analysis of the WRIC for finite Gaussian matrices. The proposed approach relies on the exact distribution of the extreme eigenvalues for Wishart matrices or on its gamma approximation based on Tracy-Widom (TW)'s laws. In particular, we derive a tight lower bound on the cumulative distribution function (CDF) of the WRICs and on the probability of satisfying an arbitrary recovery condition, much tighter than those based on the concentration inequalities. Moreover, we propose a unified framework to quantify the recovery limits of joint sparse reconstruction algorithms with WRIC based sufficient conditions, for both noiseless and noisy measurements. More precisely, the analysis estimates the maximum support cardinality of row sparse matrices, such that a target probability of recovery is assured. Then, the unified approach is applied to theoretically assess the reconstruction through three algorithms, i.e., $\ell_{2,1}$, SA-Music, and OSMP. Finally, we provide sufficient conditions in terms of the asymmetric WRICs permitting recovery with higher $s$, compared to those obtained through the symmetric WRIC.

Throughout the letter, $|\cdot|$ denotes the cardinality of a set, $\|\cdot\|$ indicates the $\ell_{2}$-norm of a vector or the spectral norm of a matrix, $\mathbf{a}_{k}$ is the $k$ th column of a matrix $\mathbf{A}$, $P(a, x)=1 / \Gamma(a) \int_{0}^{x} t^{a-1} e^{-t} d t$ is the regularized lower incomplete gamma function, $(\cdot)^{\dagger}$ is the pseudoinverse, and $\mathbf{P}_{x}$ denotes the projection matrix on the subspace $X$.

\section{WRIC AND EIGENVALUES STATistics}

In this section, we describe the problem and provide the mathematical tools needed to analyze the WRICs. In particular,

\footnotetext{
${ }^{2}$ The WRIC and RIC, while apparently similar, are distinct in the definition, meaning, and provided recovery guarantee (average vs worst case) [1], [6].
} 
a generalization of the WRIC considering lower and upper asymmetric constants is reported below.

Definition 1 (The asymmetric WRICs [6], [18]). Let A be an $m \times n$ matrix, $S \subset \Omega \triangleq\{1,2, \ldots, n\}$ be a set of indexes with $|S|=s$, the lower WRIC (LWRIC) and upper WRIC (UWRIC), $\underline{\delta}_{r}(\mathbf{A}, S)$ and $\bar{\delta}_{r}(\mathbf{A}, S)$, are the smallest constants such that the inequalities

$$
1-\underline{\delta}_{r}(\mathbf{A}, S) \leq \frac{\|\mathbf{A} \mathbf{v}\|^{2}}{\|\mathbf{v}\|^{2}} \leq 1+\bar{\delta}_{r}(\mathbf{A}, S)
$$

are satisfied for all $\mathbf{v} \in \mathbb{R}^{n}$ with support $S \cup T$ where $T \subset \Omega / S$ is any set with $|T| \leq r$. The symmetric WRIC defined as $\delta_{r}(\mathbf{A}, S) \triangleq \max \left\{\underline{\delta}_{r}(\mathbf{A}, S), \bar{\delta}_{r}(\mathbf{A}, S)\right\}$ simultaneously satisfies both inequalities.

We aim to upper bound the WRICs of Gaussian measurement matrices, so that joint sparse recovery is guaranteed with predefined probability. Hence, the statistics of WRICs should be calculated. Let us start from the Rayleigh quotient inequality for the ratio in (3), for a fixed set $T$

$$
\lambda_{\min }\left(\mathbf{A}_{Z}^{T} \mathbf{A}_{Z}\right) \leq \frac{\left\|\mathbf{A}_{Z} \mathbf{v}_{Z}\right\|^{2}}{\|\mathbf{v}\|^{2}} \leq \lambda_{\max }\left(\mathbf{A}_{Z}^{T} \mathbf{A}_{Z}\right)
$$

where $Z \triangleq S \cup T, \mathbf{A}_{Z}$ is the $m \times z$ submatrix with $z \triangleq|Z|=$ $s+r$, and $\lambda_{\min }\left(\mathbf{A}_{Z}^{T} \mathbf{A}_{Z}\right)$ and $\lambda_{\max }\left(\mathbf{A}_{Z}^{T} \mathbf{A}_{Z}\right)$ are the minimum and maximum eigenvalues of the Wishart matrix, respectively. Therefore, from (4) and accounting for all possible sets of $T$, the asymmetric WRICs can be

$$
\begin{aligned}
& \underline{\delta}_{r}(\mathbf{A}, S)=1-\min _{T,|T|=r} \lambda_{\min }\left(\mathbf{A}_{Z}^{T} \mathbf{A}_{Z}\right) \\
& \bar{\delta}_{r}(\mathbf{A}, S)=\max _{T,|T|=r} \lambda_{\max }\left(\mathbf{A}_{Z}^{T} \mathbf{A}_{Z}\right)-1 .
\end{aligned}
$$

The WRICs of Gaussian matrices are functions of the extreme eigenvalues of the corresponding Wishart matrices, and thus are themselves random variables (r.v.s).

In order to study the exact distribution of extreme eigenvalues we use the framework developed in [32]. Hereafter, we consider $\mathbf{A}$ with i.i.d. entries drawn from a zero-mean Gaussian distribution with variance $1 / \mathrm{m}$. The exact CDFs of the extreme eigenvalues of $\mathbf{A}_{Z}^{T} \mathbf{A}_{Z}$ are calculated as

$$
\begin{aligned}
& \mathbb{P}\left\{\lambda_{\min }\left(\mathbf{A}_{Z}^{T} \mathbf{A}_{Z}\right) \leq x\right\}=1-\psi(m x, \infty) \\
& \mathbb{P}\left\{\lambda_{\max }\left(\mathbf{A}_{Z}^{T} \mathbf{A}_{Z}\right) \leq x\right\}=\psi(0, m x)
\end{aligned}
$$

where $\psi(a, b)$ is the probability that all eigenvalues of a Wishart matrix are within the interval $(a, b)$, computed by Algorithm 1 in [32]. The exact expressions (7) and (8) are computationally easy for moderate matrix dimensions (we used them up to $m=2 \cdot 10^{4}$ ). For larger dimensions, the exact CDFs can be approximated, based on TW's laws [33], by shifted and scaled gamma distributions [32]

$$
\begin{aligned}
& \mathbb{P}\{\left.\lambda_{\min }\left(\mathbf{A}_{Z}^{T} \mathbf{A}_{Z}\right) \leq x\right\} \\
& \simeq \tilde{\psi}_{\text {lower }}(x) \triangleq 1-P\left(k, \frac{(\alpha-(\ln (m x)-v) / \tau)^{+}}{\theta}\right) \\
& \mathbb{P}\left\{\lambda_{\max }\left(\mathbf{A}_{Z}^{T} \mathbf{A}_{Z}\right) \leq x\right\} \\
& \simeq \tilde{\psi}_{\text {upper }}(x) \triangleq P\left(k, \frac{(\alpha+(m x-\mu) / \sigma)^{+}}{\theta}\right)
\end{aligned}
$$

where $k=46.446, \theta=0.186, \alpha=9.848, \mu=(\sqrt{m}+\sqrt{z})^{2}$, $\sigma=\sqrt{\mu}(1 / \sqrt{z}+1 / \sqrt{m})^{1 / 3}, x^{+}=\max \{x, 0\}$, and

$$
\begin{aligned}
& \tau=\frac{\left((z-1 / 2)^{-1 / 2}-(m-1 / 2)^{-1 / 2}\right)^{1 / 3}}{\sqrt{m-1 / 2}-\sqrt{z-1 / 2}} \\
& v=2 \ln (\sqrt{m-1 / 2}-\sqrt{z-1 / 2})+\tau^{2} / 8 .
\end{aligned}
$$

Alternatively, since the TW distribution can also be approximated by its asymptotic tail expansion [34], $P(k, z)$ in (9) and (10) can be replaced by

$$
\widetilde{P}(z) \triangleq 1-\frac{1}{4 \sqrt{\pi}}(z \theta-\alpha)^{\frac{3}{4}} e^{-\frac{2}{3}(z \theta-\alpha)^{\frac{3}{2}}} \quad \text { for } z>\frac{\alpha}{\theta}
$$

whenever simpler expressions for WRICs are preferable.

It can be verified from the exact distributions that the extreme eigenvalues asymmetrically deviate from unity. Hence, the asymmetric analysis leads to more accurate description of the WRICs, as previously noticed for the RIC in [7]-[12].

\section{WRIC ANALYSIS FOR GAUSSIAN MATRICES}

We derive at first lower bounds on the CDFs of the WRICs using the exact distributions of the extreme eigenvalues and their gamma approximation. Then, we deduce the WRIC thresholds which are not exceeded with a target probability.

Specifically, the CDF of the LWRIC is lower bounded from (5) and (7) $\mathrm{by}^{3}$

$$
\begin{aligned}
F_{\text {LWRIC }}(x) & \triangleq 1-\left(\begin{array}{c}
n-s \\
r
\end{array}\right)[1-\psi(m(1-x), \infty)] \\
& \simeq 1-\left(\begin{array}{c}
n-s \\
r
\end{array}\right) \tilde{\psi}_{\text {lower }}(1-x)
\end{aligned}
$$

where the binomial coefficient is from the union bound to account for all possible sets $T$ with $|T|=r$, and (11b) is based on (9). Let us define the LWRIC threshold, $\underline{\delta}_{r}^{*}(s, \epsilon)$, such that

$$
\mathbb{P}\left\{\underline{\delta}_{r}(\mathbf{A}, s) \leq \underline{\delta}_{r}^{*}(s, \epsilon)\right\} \geq 1-\epsilon
$$

is satisfied. Then, from (11) and (12), it can be estimated as

$$
\begin{aligned}
& \underline{\delta}_{r}^{*}(s, \epsilon)=\psi_{m z, \text { lower }}^{-1}\left(1-\frac{\epsilon}{\left(\begin{array}{c}
n-s \\
r
\end{array}\right)}\right) \\
\simeq & 1-\frac{1}{m} \exp \left(\tau\left[\alpha-\theta P^{-1}\left(k, 1-\frac{\epsilon}{\left(\begin{array}{c}
n-s \\
r
\end{array}\right)}\right)\right]+v\right)
\end{aligned}
$$

where $\psi_{\text {lower }}^{-1}(y)$ is the inverse of $\psi(m(1-x), \infty)$.

Similarly, the CDF of the UWRIC is lower bounded by

$$
\begin{aligned}
F_{\text {UWRIC }}(x) & \triangleq 1-\left(\begin{array}{c}
n-s \\
r
\end{array}\right)[1-\psi(0, m(1+x))] \\
& \simeq 1-\left(\begin{array}{c}
n-s \\
r
\end{array}\right)\left[1-\tilde{\psi}_{\text {upper }}(1+x)\right] .
\end{aligned}
$$

Accordingly, the UWRIC threshold is

$$
\begin{aligned}
& \bar{\delta}_{r}^{*}(s, \epsilon)=\psi_{m z, \text { upper }}^{-1}\left(1-\frac{\epsilon}{\left(\begin{array}{c}
n-s \\
r
\end{array}\right)}\right)-1 \\
& \quad \simeq \frac{\sigma}{m}\left[\theta P^{-1}\left(k, 1-\frac{\epsilon}{\left(\begin{array}{c}
n-s \\
r
\end{array}\right)}\right)-\alpha\right]+\frac{\mu}{m}-1
\end{aligned}
$$

\footnotetext{
${ }^{3}$ The set $S$ is replaced by $s$ in the notation, as the WRICs of i.i.d. random matrices statistically depend on the cardinality rather than the set itself.
} 
where $\psi_{m z \text {,upper }}^{-1}(y)$ is the inverse of $\psi(0, m(1+x))$.

Analogously, the CDF of the symmetric WRIC can be lower bounded by

$$
\begin{aligned}
& F_{\text {WRIC }}(x) \triangleq 1-\left(\begin{array}{c}
n-s \\
r
\end{array}\right)[1-\psi(m(1-x), m(1+x))] \\
& \simeq 1-\left(\begin{array}{c}
n-s \\
r
\end{array}\right)\left[1+\tilde{\psi}_{\text {lower }}(1-x)-\tilde{\psi}_{\text {upper }}(1+x)\right]
\end{aligned}
$$

where for (16b) we applied the union bound to $1-\psi(a, b)$. Following the same reasoning, the symmetric WRIC threshold can be derived as $\delta_{r}^{*}(s, \epsilon)=F_{\mathrm{WRIC}}^{-1}(\epsilon)$.

\section{UNIFIEd FrAmEWORK FOR RECOVERY ASSESSMENT}

In this section, the WRIC thresholds are used to quantify the maximum $s$, denoted $\widehat{s}$, permitting recovery with a target probability. Let us consider the recovery of a random row sparse matrix $\mathbf{X}$ with support cardinality $s$, acquired through a random measurement matrix $\mathbf{A}$. In general, for a given $\mathbf{A}$, if a sufficient condition stated usually in the form [6], [17]-[19]

$$
f_{\mathrm{c}}\left(\underline{\delta}_{1}(\mathbf{A}, s), \bar{\delta}_{1}(\mathbf{A}, s)\right)<1
$$

is fulfilled, then recovery is guaranteed with probability $P_{\mathrm{r} \mid \mathrm{c}}$ depending on the distribution of $\mathbf{X} .{ }^{4}$ Since $\mathbf{A}$ is random, the sufficient condition (17) is satisfied with some probability $P_{\mathrm{c}}^{\mathbf{A}}$, and recovery is ensured with probability at least

$$
P_{\mathrm{r}}=P_{\mathrm{r} \mid \mathrm{c}} P_{\mathrm{c}}^{\mathrm{A}} \text {. }
$$

In order to find $\widehat{s}$ satisfying $P_{\mathrm{r}} \geq \eta$ for a given $P_{\mathrm{r} \mid \mathrm{c}}$, we need to ensure that $P_{\mathrm{c}}^{\mathrm{A}} \geq \eta / P_{\mathrm{r} \mid \mathrm{c}}$. Hence, we propose to substitute the WRICs in (17) with the WRIC thresholds (13) and (15). Then, $\widehat{s}$ can be calculated as the maximum $s$ compatible with $f_{\mathrm{c}}\left(\underline{\delta}_{1}^{*}(\mathbf{A}, s, \epsilon), \bar{\delta}_{1}^{*}(\mathbf{A}, s, \epsilon)\right)<1$, where $\epsilon$ is derived to meet the required $P_{\mathrm{c}}^{\mathrm{A}}$. By exploiting the monotonicity of $f_{\mathrm{c}}(\cdot, \cdot)$ and the union bound, $1-P_{\mathrm{c}}^{\mathrm{A}}$ can be upper bounded by

$$
\begin{aligned}
& \mathbb{P}\left\{f_{\mathrm{c}}\left(\underline{\delta}_{1}(\mathbf{A}, \widehat{s}), \bar{\delta}_{1}(\mathbf{A}, \widehat{s})\right) \geq f_{\mathrm{c}}\left(\underline{\delta}_{1}^{*}(\widehat{s}, \epsilon), \bar{\delta}_{1}^{*}(\widehat{s}, \epsilon)\right)\right\} \\
& \leq \mathbb{P}\left\{\underline{\delta}_{1}(\mathbf{A}, \widehat{s}) \geq \underline{\delta}_{1}^{*}(\widehat{s}, \epsilon)\right\}+\mathbb{P}\left\{\bar{\delta}_{1}(\mathbf{A}, \widehat{s}) \geq \bar{\delta}_{1}^{*}(\widehat{s}, \epsilon)\right\} \leq 2 \epsilon .
\end{aligned}
$$

So the goal $P_{\mathrm{r}} \geq \eta$ is fulfilled for

$$
\epsilon=\epsilon\left(\eta, P_{\mathrm{r} \mid \mathrm{c}}\right)=1 / 2-\eta /\left(2 P_{\mathrm{r} \mid \mathrm{c}}\right) .
$$

In the following, we investigate the performance of three reconstruction methods (i.e., $\ell_{2,1}$-minimization, SA-Music, and OSMP), when $\mathbf{X}$ is a sparse Gaussian matrix and $\mathbf{A}$ is Gaussian with normalized columns [17]-[19]. ${ }^{5}$

\section{A. Perfect Recovery from Noiseless Measurements}

The first example considers the reconstruction via $\ell_{2,1^{-}}$ minimization program, for which it has been proved that if

$$
\left\|\mathbf{A}_{S}^{\dagger} \mathbf{a}_{k}\right\|<\alpha<1, \quad \forall k \notin S
$$

\footnotetext{
${ }^{4}$ Note that $f_{\mathrm{c}}\left(\underline{\delta}_{1}, \bar{\delta}_{1}\right)$ is a non-decreasing function in both $\underline{\delta}_{1}$ and $\bar{\delta}_{1}$.

${ }^{5}$ For large $m$, the $\ell_{2}$-norm of each column of $\mathbf{A}$ is approximately one.
}

then the matrix $\mathbf{X}$ can be recovered via (2) with probability at least $P_{\mathrm{r} \mid \mathrm{c}}(\alpha) \triangleq 1-n \exp \left(-\ell\left(\alpha^{-2}+2 \log \alpha\right) / 2-1\right)$ [17]. Condition (20) is expressed in terms of the WRIC as [17]

$$
\frac{\delta_{1}(\mathbf{A}, s)}{1-\delta_{1}(\mathbf{A}, s)}<\alpha \text {. }
$$

We propose relaxing condition (21) to a milder one (i.e., easily satisfied for larger $s$ ). Applying properties of the spectral norm to (20) and considering (5), we get

$$
\begin{gathered}
\left\|\mathbf{A}_{S}^{\dagger} \mathbf{a}_{k}\right\| \leq \delta_{1}(\mathbf{A}, s)\left\|\left(\mathbf{A}_{S}^{*} \mathbf{A}_{S}\right)^{-1}\right\| \leq \frac{\delta_{1}(\mathbf{A}, s)}{\lambda_{\min }\left(\mathbf{A}_{S}^{*} \mathbf{A}_{S}\right)} \\
\leq \frac{\delta_{1}(\mathbf{A}, s)}{1-\underline{\delta}_{0}(\mathbf{A}, s)}<\alpha .
\end{gathered}
$$

Since $\underline{\delta}_{0}(\mathbf{A}, s) \leq \underline{\delta}_{1}(\mathbf{A}, s) \leq \delta_{1}(\mathbf{A}, s)$, the left hand side of (22) is less than that of (21), leading to higher estimates of $\widehat{s}$.

Now, following the outlined procedure, the maximum $s$ satisfying the sufficient condition

$$
\frac{\delta_{1}^{*}\left(\mathbf{A}, s, \epsilon\left(\eta, P_{\mathrm{r} \mid \mathrm{c}}(\alpha)\right)\right)}{1-\underline{\delta}_{0}^{*}\left(\mathbf{A}, s, \epsilon\left(\eta, P_{\mathrm{r} \mid \mathrm{c}}(\alpha)\right)\right)}<\alpha
$$

denoted by $\breve{s}(\alpha)$, can be found for a given $\alpha$. Finally, a tighter upper bound on the maximum support cardinality is obtained by maximizing over $\alpha$ as

$$
\widehat{s}=\max _{0<\alpha<1} \breve{s}(\alpha) .
$$

\section{B. Robust Support Estimation from Noisy Measurements}

In the presence of noisy measurements, (1) becomes

$$
\mathbf{Y}=\mathbf{A X}+\mathbf{Z}
$$

where $\mathbf{Z}$ represents the noise. Approximate recovery of $\mathbf{X}$ may consist of a joint support estimation followed by signal reconstruction through the resulting overdetermined system.

For example, the support can be estimated via SA-Music algorithm given that a sufficient condition on WRICs, on the form of (17), is satisfied [18]. More precisely, denoting the signal subspace by $X$, let $\widehat{\mathcal{R}}$ and $\widehat{X}$ be $\hat{\ell}$-dimensional subspaces of $\mathbb{R}^{m}$ and $\mathcal{X}$, respectively, with $\left\|\mathbf{P}_{\widehat{\mathcal{R}}}-\mathbf{P}_{\widehat{X}}\right\| \leq \kappa{ }^{6}$ Then, SA-Music applied to $\widehat{\mathcal{R}}$ recovers the support if

$$
\sqrt{\frac{1-\underline{\delta}_{1}(\mathbf{A}, s)}{1+\bar{\delta}_{1}(\mathbf{A}, s)}} \frac{\sqrt{\hat{\ell} / s} \sqrt{1-\underline{\delta}_{1}(\mathbf{A}, s)}-\sqrt{\underline{\delta}_{1}(\mathbf{A}, s)}}{2+\sqrt{\hat{\ell} / s} \sqrt{1-\underline{\delta}_{1}(\mathbf{A}, s)}-\sqrt{\underline{\delta}_{1}(\mathbf{A}, s)}}>\kappa \text {. }
$$

Alternatively, if support estimation is performed via OSMP, the sufficient condition is [19]

$$
\begin{aligned}
& \underline{\delta}_{1}(\mathbf{A}, s)<1-\max \{4 \kappa(1-\kappa), \\
& \left.\quad\left[\frac{1}{1+\hat{\ell} / s}\left(2 \kappa \sqrt{\hat{\ell} / s}+\sqrt{1+\hat{\ell} / s-4 \kappa^{2}}\right)\right]^{2}\right\} .
\end{aligned}
$$

Note that the probability of exact support recovery via SA-Music and OSMP is greater than the probability of satisfying (24) and (25), respectively (i.e., $P_{\mathrm{r} \mid \mathrm{c}}=1$ ). We would like

\footnotetext{
${ }^{6}\left\|\mathbf{P}_{\widehat{\mathcal{R}}}-\mathbf{P}_{\widehat{x}}\right\|$ is bounded with high probability for Gaussian noise [18].
} 


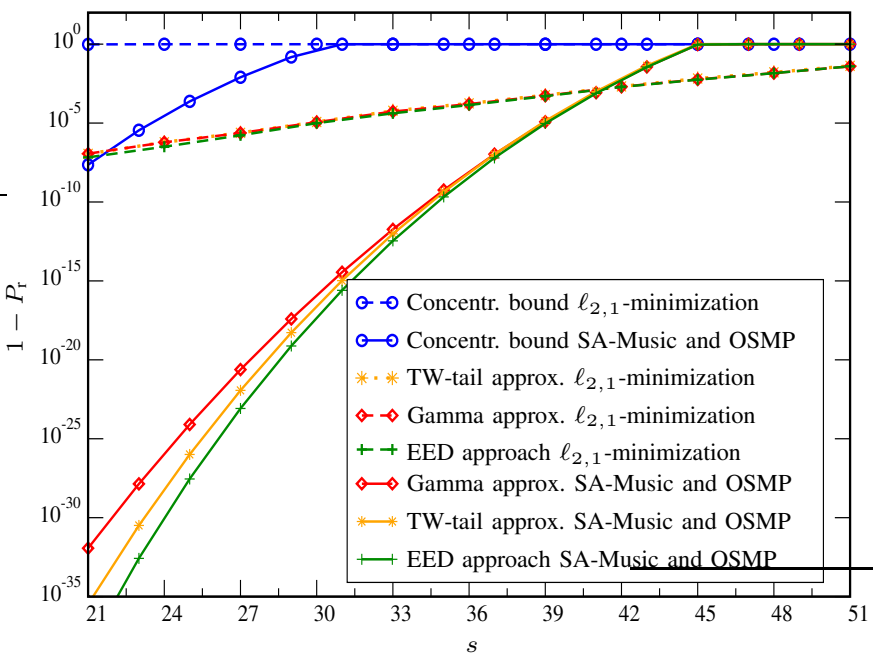

Fig. 1. Upper bounds on the probability of recovery failure using the proposed approaches and concentration bound for various algorithms, with $n=2 m=6000, \ell=20$, and $\kappa=0$.

to note also that (24) and (25) relax the recovery conditions in [18] and [19] by utilizing the asymmetric WRICs. The original formulas in [18] and [19] can be obtained by substituting $\underline{\delta}_{1}(\mathbf{A}, s)$ and $\bar{\delta}_{1}(\mathbf{A}, s)$ with $\delta_{1}(\mathbf{A}, s)$.

\section{Numerical Results}

In this section, numerical results are presented to investigate the WRICs and the estimated maximum joint support cardinality for various recovery algorithms. Note that the analysis based on the exact extreme eigenvalue statistics (7) and (8) will be referred as the exact eigenvalues distribution (EED).

Fig. 1 shows upper bounds on the probability of reconstruction failure derived from the concentration of measure in [18, Proposition 6.1], along with the EED bound and gamma and TW tail expansion based approximations. The analysis is based on the sufficient recovery conditions via SA-Music and OSMP and via $\ell_{2,1}$-minimization (21). It results that the proposed methods provide tighter bounds compared to the concentration inequalities (many orders of magnitude). Also, the gamma and TW approximations well describe the exact eigenvalues based analysis (overlapped for $1-P_{\mathrm{r}} \geq 10^{-10}$ ). Moreover, the theoretical performance of SA-Music and OSMP are coincident, as they have the same condition for the noiseless case. ${ }^{7}$ Additionally, they have better performance for small $s$, while the $\ell_{2,1}$-minimization is superior at higher $s$.

Fig. 2 plots the contours of the UWRIC thresholds calculated by means of the EED and gamma approximation (15). As can be seen the gamma approximation is quite accurate, and provides tight upper bounds on the UWRIC thresholds for small values, relevant to sparse recovery.

Finally, the normalized maximum joint support cardinality, $\widehat{s} / m$, for various recovery algorithms is shown in Fig 3. In particular, $\widehat{s}$ has been estimated using the associated asymmetric and symmetric conditions, where the WRICs are calculated by the EED, for various noise parameters $\kappa$ and probability of correct support estimation at least $0.999 .{ }^{8}$ As can be noticed,

\footnotetext{
${ }^{7}$ Note that both the conditions in (24) and (25) reduce to $\delta_{1}(\mathbf{A}, s) \leq \ell /(s+\ell)$ in the noiseless case $(\kappa=0, \hat{\ell}=\ell)$.

${ }^{8}$ Support estimation is sufficient for perfect recovery in the noiseless case.
}

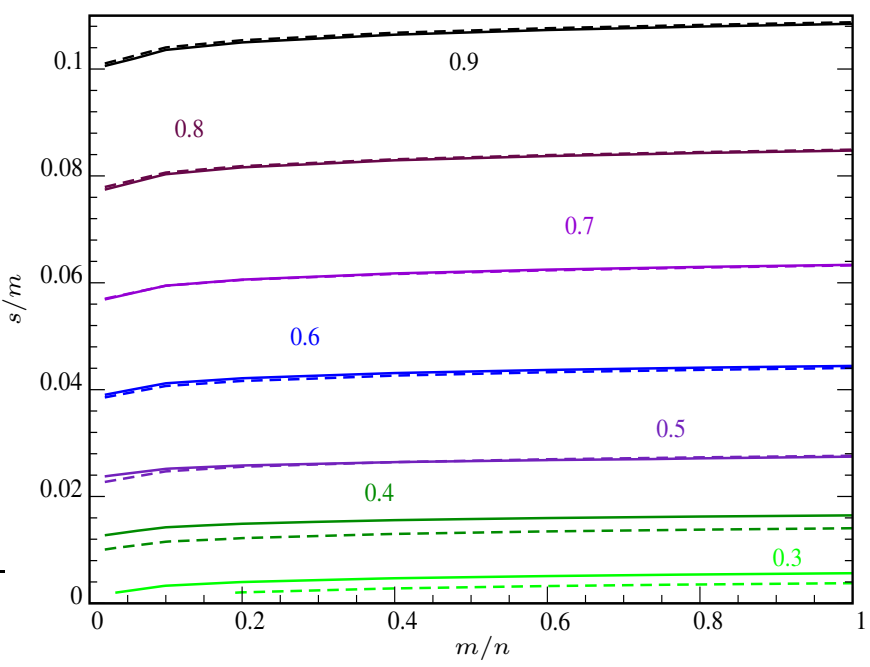

Fig. 2. Level sets of the UWRIC threshold $\bar{\delta}_{1}\left(\mathbf{A}, s, 10^{-3}\right) \in\{0.3, \ldots, 0.9\}$ using the EED (solid) and gamma approximation (dashed), for $m=3000$.

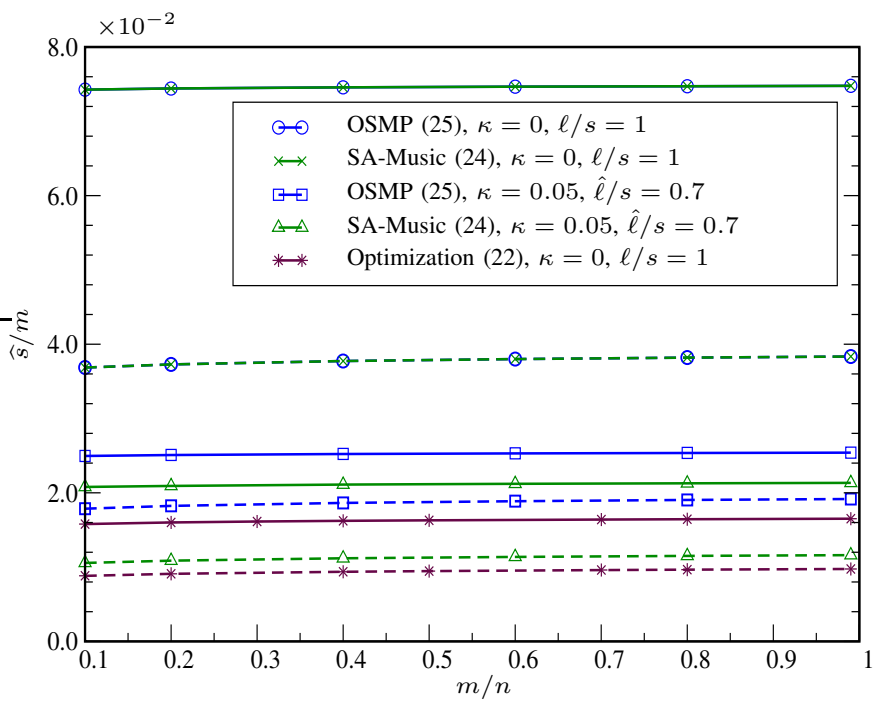

Fig. 3. The normalized $\widehat{s}$ via the three recovery methods through asymmetric (solid) and symmetric (dashed) conditions, with $P_{\mathrm{r}} \geq 0.999$ and $m=3000$.

OSMP allows recovery of less sparse signals, compared to SA-Music, for noisy measurements. In the noiseless case, for the set of chosen parameters, the mixed norm minimization indicates lower $\widehat{s}$. Additionally, it is evident that the proposed asymmetric conditions provide higher estimates of $\widehat{s}$ compared to the symmetric ones (up to $100 \%$ increase in $s$ ).

\section{CONCLUSION}

We have studied the weak restricted isometry constant of Gaussian matrices, which is particularly important for MMV problem arising in many applications, e.g., localization, imaging, and multivariate regression. The proposed analysis provides tighter bounds on the probability of recovery, several orders of magnitude compared to the concentration bounds. Additionally, the suggested framework allows comparing various joint sparse recovery algorithms in terms of the maximum allowable support cardinality. Finally, the analysis indicates that the derived asymmetric WRIC based conditions are preferable over those based on WRIC, as they result in better performance bounds. 


\section{REFERENCES}

[1] E. Candes and T. Tao, "Decoding by linear programming," IEEE Trans. Inf. Theory, vol. 51, no. 12, pp. 4203-4215, Dec. 2005.

[2] D. Donoho, "Compressed sensing," IEEE Trans. Inf. Theory, vol. 52, no. 4, pp. 1289-1306, April 2006.

[3] _ _ "For most large underdetermined systems of linear equations the minimal $\ell_{1}$-norm solution is also the sparsest solution," Comm. Pure Appl. Math., vol. 59, no. 6, pp. 797-829, 2006.

[4] E. Candes and T. Tao, "Near-optimal signal recovery from random projections: Universal encoding strategies?" IEEE Trans. Inf. Theory, vol. 52 , no. 12 , pp. 5406-5425, Dec. 2006.

[5] D. Needell and R. Vershynin, "Signal recovery from incomplete and inaccurate measurements via regularized orthogonal matching pursuit," IEEE J. Sel. Topics Signal Process., vol. 4, no. 2, pp. 310-316, April 2010.

[6] E. Candes and Y. Plan, "A probabilistic and RIPless theory of compressed sensing," IEEE Trans. Inf. Theory, vol. 57, no. 11, pp. 72357254, Nov. 2011.

[7] J. Blanchard, C. Cartis, and J. Tanner, "Compressed sensing: How sharp is the restricted isometry property?" SIAM review, vol. 53, no. 1, pp. 105-125, 2011.

[8] B. Bah and J. Tanner, "Improved bounds on restricted isometry constants for Gaussian matrices," SIAM J. Matrix Anal. Appl., vol. 31, no. 5, pp. 2882-2898, 2010

[9] J. Blanchard, C. Cartis, J. Tanner, and A. Thompson, "Phase transitions for greedy sparse approximation algorithms," Appl. Comput. Harmon. Anal., vol. 30, no. 2, pp. 188-203, 2011.

[10] M. Chiani, A. Elzanaty, and A. Giorgetti, "Analysis of the restricted isometry property for Gaussian random matrices," in Proc. IEEE Int. Conf. on Global Comm. (GLOBECOM), San Diego, USA, Dec. 2015 , pp. 1-6.

[11] A. Elzanaty, A. Giorgetti, and M. Chiani, "On sparse recovery using finite Gaussian matrices: RIP-based analysis," in Proc. IEEE Stati. Signal Processing Work. (SSP), June 2016, pp. 395-399.

[12] S. Foucart and M.-J. Lai, "Sparsest solutions of underdetermined linear systems via $\ell_{q}$-minimization for $0<q \leq 1$," Appl. Comput. Harmon. Anal., vol. 26, no. 3, pp. $395-407,2009$.

[13] W. Bajwa, J. Haupt, A. Sayeed, and R. Nowak, "Compressed channel sensing: A new approach to estimating sparse multipath channels," Proc. IEEE, vol. 98, no. 6, pp. 1058-1076, June 2010.

[14] M. Duarte, S. Sarvotham, D. Baron, M. Wakin, and R. Baraniuk, "Distributed compressed sensing of jointly sparse signals," in Proc. Thirty-Ninth Asilomar Conf. on Signals, Systems and Comp., Oct. 2005, pp. 1537-1541.

[15] J. Tropp, "Algorithms for simultaneous sparse approximation. Part II: Convex relaxation," Signal Processing, vol. 86, no. 3, pp. 589-602, March 2006

[16] J. Chen and X. Huo, "Theoretical results on sparse representations of multiple-measurement vectors," IEEE Trans. Signal Process., vol. 54 no. 12, pp. 4634-4643, Dec. 2006.
[17] Y. Eldar and H. Rauhut, "Average case analysis of multichannel sparse recovery using convex relaxation," IEEE Trans. Inf. Theory, vol. 56 , no. 1, pp. 505-519, Jan. 2010.

[18] K. Lee, Y. Bresler, and M. Junge, "Subspace methods for joint sparse recovery," IEEE Trans. Inf. Theory, vol. 58, no. 6, pp. 3613-3641, June 2012.

[19] K. Kim and S. Chung, "Greedy subspace pursuit for joint sparse recovery," arXiv preprint arXiv:1601.07087, Jan 2016.

[20] S. Cotter, B. Rao, K. Engan, and K. Kreutz-Delgado, "Sparse solutions to linear inverse problems with multiple measurement vectors," IEEE Trans. Signal Process., vol. 53, no. 7, pp. 2477-2488, July 2005.

[21] R. Gribonval, H. Rauhut, K. Schnass, and P. Vandergheynst, "Atoms of all channels, unite! average case analysis of multi-channel sparse recovery using greedy algorithms," J. Fourier Anal. Appl., vol. 14, no. 5, pp. $655-687$, 'Oct. 2008.

[22] J. Tropp, A. Gilbert, and M. Strauss, "Algorithms for simultaneous sparse approximation. Part I: Greedy pursuit,” Signal Processing, vol. 86, no. 3, pp. 572-588, March 2006.

[23] J. Blanchard, M. Cermak, D. Hanle, and Y. Jing, "Greedy algorithms for joint sparse recovery," IEEE Trans. Signal Process., vol. 62, no. 7, pp. 1694-1704, Apr. 2014

[24] J. Blanchard, J. Tanner, and K. Wei, "Conjugate gradient iterative hard thresholding: Observed noise stability for compressed sensing," IEEE Trans. Signal Process., vol. 63, no. 2, pp. 528-537, Jan 2015.

[25] M. Davies and Y. Eldar, "Rank awareness in joint sparse recovery," IEEE Trans. Inf. Theory, vol. 58, no. 2, pp. 1135-1146, Feb. 2012.

[26] R. Baraniuk, V. Cevher, M. Duarte, and C. Hegde, "Model-based compressive sensing," IEEE Trans. Inf. Theory, vol. 56, no. 4, pp. 1982 2001, April 2010

[27] S. Gogineni and A. Nehorai, "Target estimation using sparse modeling for distributed MIMO radar," IEEE Trans. Signal Process., vol. 59, no. 11 , pp. 5315-5325, Nov. 2011.

[28] D. Ge, X. Jiang, and Y. Ye, "A note on the complexity of $\ell_{p}$ minimization," Math. Program., vol. 129, no. 2, pp. 285-299, Oct. 2011.

[29] R. Schmidt, "Multiple emitter location and signal parameter estimation," IEEE Trans. Antennas Propag., vol. 34, no. 3, pp. 276-280, Mar. 1986.

[30] P. Feng and Y. Bresler, "Spectrum-blind minimum-rate sampling and reconstruction of multiband signals," in Proc IEEE Int. Conf. on Acoustics, Speech, and Signal Process., vol. 3, May 1996, pp. 1688-1691.

[31] A. Edelman, "Eigenvalues and condition numbers of random matrices," SIAM J. Matrix Anal. Appl., vol. 9, no. 4, pp. 543-560, 1988.

[32] M. Chiani, "On the probability that all eigenvalues of Gaussian and Wishart random matrices lie within an interval," IEEE Trans. Inf. Theory, April 2017, Preprint.

[33] M. Johnstone, "On the distribution of the largest eigenvalue in principal components analysis," The Annals of Statistics, vol. 29, no. 2, pp. 295$327,2001$.

[34] G. Borot and C. Nadal, "Right tail asymptotic expansion of TracyWidom beta laws," Random Matrices: Theory and Applications, vol. 1, no. 3, pp. 1-23, Jul. 2012. 